\title{
Sonoluminescence as the PeTa Radiation, Part Two
}

\author{
Vitali A. Tatartchenko \\ CNRS, Aix-Marceille University, Provence, France \\ Email: vitali.tatartchenko@orange.fr
}

How to cite this paper: Tatartchenko, V.A. (2017) Sonoluminescence as the PeTa Radiation, Part Two. Optics and Photonics Journal, 7, 197-220.

https://doi.org/10.4236/opj.2017.711019

Received: October 6, 2017

Accepted: November 4, 2017

Published: November 7, 2017

Copyright $\odot 2017$ by author and Scientific Research Publishing Inc. This work is licensed under the Creative Commons Attribution International License (CC BY 4.0).

http://creativecommons.org/licenses/by/4.0/

\begin{abstract}
This paper is a continuation of one published in this journal nine months ago. The two papers present a model of cavitational luminescence (CL), multi-bubble sonoluminescence (MBSL), one-bubble sonoluminescence (OBSL), and laser-induced bubble luminescence (LIBL). The basis of this model is the PeTa (Perel'manTatartchenko) effect, a nonequilibrium characteristic radiation under first-order phase transitions, especially vapour condensation. In this model, the main role is given to the liquid, where the evaporation, condensation, flash, and subsequent collapse of bubbles occur. The instantaneous vapour condensation inside the bubble is a reason for the CL/MBSL/OBSL/LIBL. Apparently, the dissolved gases and other impurities in the liquid are responsible for peaks that appear at the background of the main spectrum. They are most likely excited by a shock wave occurred during the collapse. This paper, in contrast to the previous one, presents a slightly expanded model that explains additional experimental data concerning especially the LIBL spectrum. As a result, today we are not aware of any experimental data that would contradict the PeTa model, and we continue to assert that there is no mystery to the CL/MBSL/OBSL/LIBL phenomena, as well as no reason to hope that they can be used for high-temperature chemical reactions, and even more so for a thermonuclear ones.
\end{abstract}

\section{Keywords}

Cavitational Luminescence (CL), Sonoluminescence (SL), Multi-Bubble Sonoluminescence (MBSL), One-Bubble Sonoluminescence (OBSL), Laser-Induced Bubble Luminescence (LIBL), PeTa (Perel'man-Tatartchenko)

Effect, First-Order Phase Transitions, Evaporation, Condensation

\section{Introduction}

Since 2010, a nonequilibrium characteristic radiation under first-order phase tran- 
sitions, for instance melt crystallisation or vapour condensation/deposition, has been called the PeTa (Perel'man-Tatartchenko) effect [1]. On the basis of this effect, in paper [2], we developed the model of cavitational luminescence (CL), and sonoluminescence (SL), multi-bubble sonoluminescence (MBSL), and one-bubble sonoluminescence (OBSL). It should be noted that even 10 years ago we proposed to consider the PeTa effect as the basis of MBSL [3] [4] [5]. But then it was done without a detailed analysis and concerned only infrared radiation under MBSL described in paper [6]. In [2], the CL/MBSL/OBSL model based on the PeTa effect was developed in detail. It explained the main experimental results. We consider this model as a first iteration of the full model. In the future, we intend to improve the model. But even now, we did not use all possibilities of the model; it is able to explain additional features of such phenomena. This especially true of laser-induced bubble luminescence (LIBL), in which vapour bubbles form in the liquid due to a short laser pulse. Interesting peculiarities in the LIBL spectrum are explained on the basis of this model, additional capabilities of which are presented below.

\section{Laser-Induced Bubble Luminescence}

\subsection{General Description}

We consider the experiments from paper [7], in which the luminescence from bubbles formed in water by short laser pulses was studied in detail. The phenomenon was first reported in paper [8]. In [7] it is called laser-induced bubble luminescence (LIBL). The main parameters of this study were the maximum bubble radius and the ambient pressure of the liquid surrounding the bubble. The dry nitrogen gas was used to pressurise the liquid. The luminescence pulse was recorded using a very fast photomultiplier tube (PMT). Two flashes are visible in Figure 1(a). With respect to the author's opinion, the first is a laser plasma flash and the second is a LIBL flash. The PeTa effect is the basis of this flash. Thus, it is impossible to call this luminescence "collapse luminescence", as the author of [7] does. This title reverses the cause and effect. From our model, luminescence is not a consequence of the bubble collapse, but collapse is a consequence of the instantaneous decrease in pressure due to condensation of the vapour. Flash occurs as a result of this condensation.

\subsection{Behaviour of a Bubble between Two Flashes}

Let us analyse the behaviour of the bubble after the first flash. Despite an evident decrease in the bubble's temperature during $\sim 100 \mu \mathrm{s}$, plasma and heated gas have sufficient initial energy to vaporise the liquid inside the bubble, leading to an increase in its size. At the end of this time, the bubble reaches its maximum size $R \approx 1 \mathrm{~mm}$ (Figure $1(\mathrm{a})$ ). We label this point number 1 . It is obvious that starting from this point, due to the continuing decrease in temperature, the energy of the matter inside the bubble is not enough to continue vaporisation of the water from the inner surface of the bubble. The size of the bubble begins to decrease. 


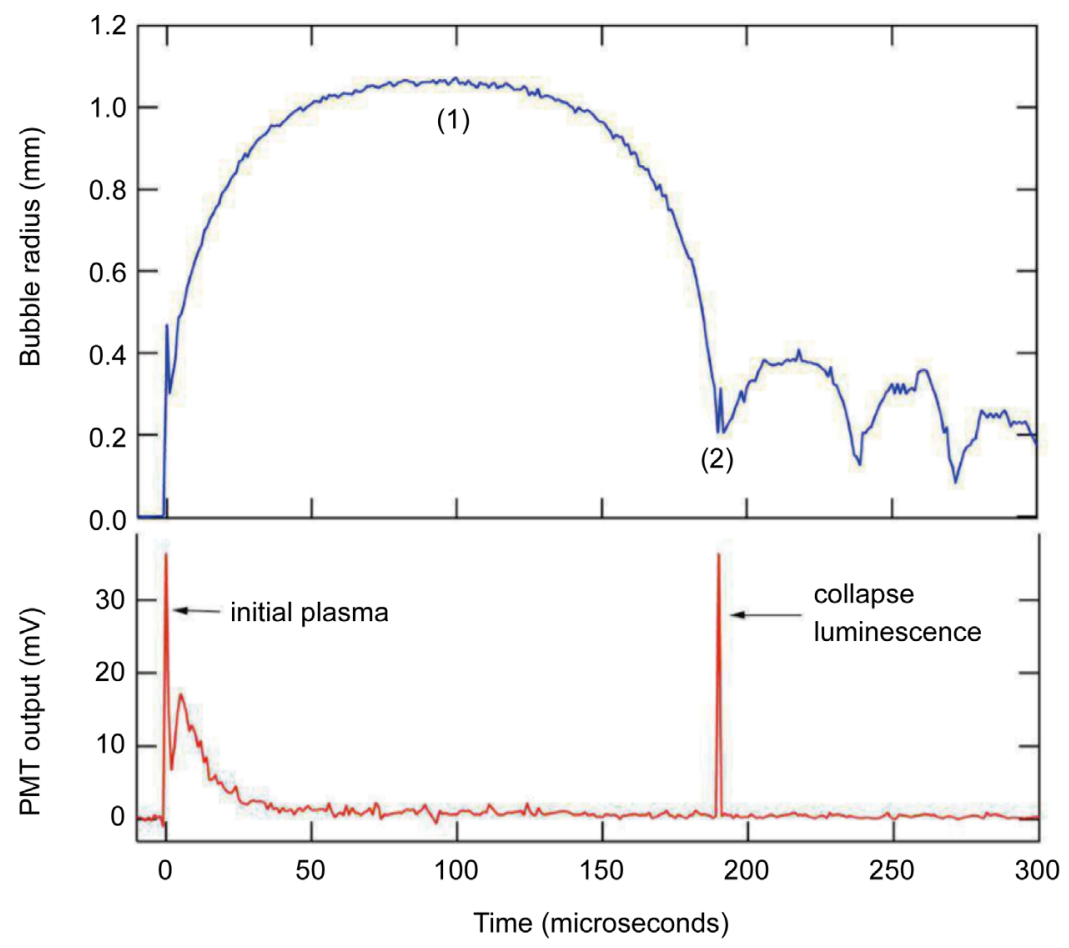

(a)

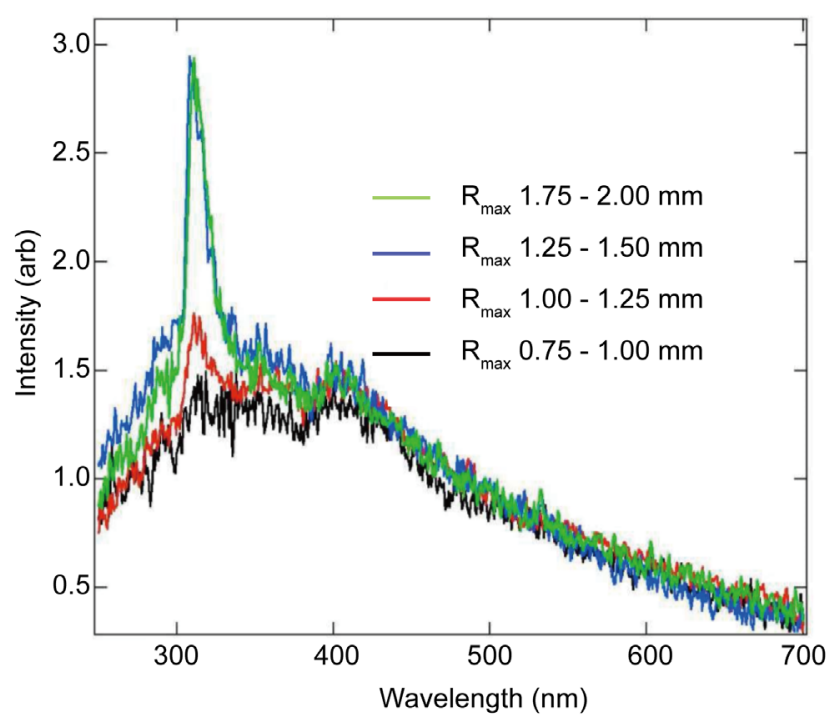

(b)

Figure 1. From Ref. [7]. (a) For LIBL, time dependence of light output recorded by PMT and bubble radius; the first flash is induced by the laser beam and the second one is the LIBL. The numbers 1 and 2 are points of the curve used in our model; (b) LIBL spectra for different comparatively large bubble radii.

This bubble behaviour is natural. Indeed, with decreasing temperature, the energy of the molecules of vapour and gas inside the bubble decreases. Consequently, the pressure within the bubble also decreases. The external pressure remains constant or even increases due to increased Laplace pressure. Thus, to preserve the mechanical balance of forces acting on the wall of the bubble, gas and vapour in- 
side the bubble should be compressed.

Let us consider the thermodynamic state of gas and vapour inside the bubble in the process of reducing its size on the basis of the analysis carried out in paper [2].

The amount of gas inside the bubble remains unchanged since the diffusion of the gas into the liquid or back will be negligibly small during the rapid change in the size of the bubble. We can assume that the behaviour of the gas inside the bubble is determined by the equation of state of an ideal gas. Consequently, the gas will contract as the bubble size decreases.

For water vapour, from Equation (14) Ref. [2], it follows that to keep the water vapour in equilibrium inside the bubble, the rate of change of the radius should not exceed $3 \mathrm{~m} \cdot \mathrm{s}^{-1}$. This value was obtained for a reasonable but arbitrary accommodation coefficient $\alpha=0.2$. The more precise analysis makes it clear that we do not need to choose an arbitrary value for $\alpha$, but it can be calculated from experiments described in paper [2]. It gives $\alpha=0.1$; thus, to maintain the equilibrium vapour pressure, the rate of change of the radius should not exceed 0.5 $\mathrm{m} \cdot \mathrm{s}^{-1}$. The bubble radius in the LIBL experiment under consideration decreases from $1 \mathrm{~mm}$ to $0.2 \mathrm{~mm}$ in $80 \mu \mathrm{s}$. Thus, the rate of change of the radius is $\sim 10$ $\mathrm{m} \cdot \mathrm{s}^{-1}$, which is 20 times higher than the maximal determined rate. As a result, the vapour inside the bubble does not have time to condense in an ordinary way, and consequently it will be compressed and supersaturated. Another factor increases supersaturation: the equilibrium vapour pressure decreases strongly with decreasing temperature.

At an elevated pressure, in supersaturated vapour, three processes occur: First, the vapour molecules are predisposed to form clusters, and most likely this occurs. Second, molecules/clusters become excited compared to the bulk liquid molecules. Third, the density of molecules/clusters in the vapour increases, and in conformity with [9], when the threshold value of this density is reached, there is an interaction of molecules and clusters through the collective radiation field. We obtain the classical situation in which PeTa radiation occurs. It is at this moment that there is an instant condensation of the vapour inside the bubble with the emission of the phase-transition energy, a subsequent decrease in pressure, a collapse of the bubble as a result of the instant pressure drop, and the formation of a shock wave. In accordance with paper [8], the subsequent changes in the size of the bubble (Figure 1(a)) are a consequence of the arrival of reflection shock waves.

\subsection{Isobaric PeTa Model of LIBL}

Let us analyse the process described above in the framework of the PeTa model. First of all, we note the essential difference between CL/SL and LIBL. In CL/SL, change in an external pressure is the driving force of the processes. In paper [2], we took into account the change in the pressure, but neglected a possible change in temperature. As a result, we used an isothermal version of the PeTa model sa- 
tisfactorily describing the process and explaining the main experimental results. In LIBL, the driving force of the process is the temperature change, so we use the isobaric version of the PeTa model, taking into account the temperature change, but neglecting a small change in pressure.

Let us analyse in detail the situation at point 1 . This point is the equilibrium one. The condition of equilibrium of forces acting on the wall of the bubble must be fulfilled at this point with respect to Equation (1), which is similar to Equation (6) from paper [2]. The only difference is the absence of a driving mechanical force $P_{D}$.

$$
P_{A 1}+P_{L 1}+P_{01}=P_{V 1}+P_{g 1}
$$

Here $P_{A 1}$ is atmospheric pressure, $P_{L 1}$ is hydrostatic pressure of the liquid pressurised by the nitrogen gas, $P_{01}$ is Laplace pressure, $P_{V 1}$ is internal partial vapour pressure, and $P_{g 1}$ is internal partial gas pressure.

Let us estimate the magnitude of the components of Equation (1): the mean value of atmospheric pressure $P_{\mathrm{A} 1} \approx 101 \mathrm{kPa}$; in accordance with the description of the experiment $P_{L 1}=101 \mathrm{kPa}$.

To determine $P_{01}$, we need to know the temperature at this point. Because it is unknown, we try to estimate the upper value of $P_{01}$. The surface tension of water increases with decreasing temperature. The maximum value of $P_{01}$ has to correspond to the minimal temperature $\sim 20^{\circ} \mathrm{C}$. Indeed, the temperature of the bubble cannot be lower than the temperature of the medium.

For $T \approx 20^{\circ} \mathrm{C}$, the water surface tension $\gamma \approx 73 \times 10^{-3} \mathrm{~N} \cdot \mathrm{m}^{-1}$. Thus, $P_{01}=2 \gamma / R_{01} \approx 15 \times 10^{-4} \mathrm{kPa}$. We can ignore a value of $P_{01}$ that is at the level of accuracy of determining other values of process parameters. It follows that it is not important what temperature is used at point 1 to determine $P_{01}$. Thus:

$$
P_{V 1}+P_{g 1} \approx 202 \mathrm{kPa}
$$

We will later return to the analysis of the situation at point 1 and use Equation (2).

Now, we will follow the further behaviour of our system. Let us consider the bubble state at point 2 , a little after the radiation flare. The radius of the bubble at this point is $0.2 \mathrm{~mm}$. Like point 1 , point 2 is also in equilibrium, and the condition of equilibrium of forces acting on the wall of the bubble must be preserved:

$$
P_{A 2}+P_{L 2}+P_{02}=P_{V 2}+P_{g 2}
$$

Let us estimate the magnitude of the components of Equation (2):

$P_{A 2}=P_{A 1} \approx 101 \mathrm{kPa} ; P_{L 2}=P_{L 1}=101 \mathrm{kPa}$. The situation with the Laplace pressure here is analogous to point 1: we assume that the temperature cannot be lower than the temperature of the surrounding fluid. Thus, $P_{01}=2 \gamma / R_{01} \approx 75 \times 10^{-4} \mathrm{kPa}$, which can be neglected, and we get:

$$
P_{V 2}+P_{g 2} \approx 202 \mathrm{kPa}
$$


Now, let us determine the temperatures at points 1 and 2 under the assumption that the mass of the gas is constant and the gas obeys the equation of state of an ideal gas. Regarding water vapour pressure, we assume that it has the equilibrium partial pressure for the respective temperatures $T_{1}$ and $T_{2}$. We get that the following temperatures satisfy all of the boundary conditions of our problem: $T_{1} \approx 120^{\circ} \mathrm{C} ; T_{2} \approx 20^{\circ} \mathrm{C}$. The partial pressures of gas and water vapour correspond to these conditions: $P_{g 1} \approx 2 \mathrm{kPa} ; P_{V 1} \approx 200 \mathrm{kPa} ; P_{g 2} \approx 200 \mathrm{kPa}$; $P_{V 2} \approx 2 \mathrm{kPa}$.

Now, let us determine how much water vapour was condensed and thus what energy was radiated during this condensation. In accordance with the arguments given above, in the process of compression of the bubble, the vapour did not condense until the PeTa effect occurred. Consequently, the mass of the vapour that took part in the PeTa process is equal to the mass difference inside the bubble at points $1\left(m_{1}\right)$ and $2\left(m_{2}\right)$. The bubble at point 1 contains $m_{1} \approx 4.7 \times 10^{-6} \mathrm{~g}$ of water vapour. The bubble at point 2 contains $m_{2} \approx 5 \times 10^{-10} \mathrm{~g}$ of water vapour. The value of $\mathrm{m}_{2}$ is beyond the accuracy of our calculations, so it can be neglected. If we assume that the entire vapour condensation energy of mass $m_{1}$ is radiated during the phase transition, then we will determine the upper limit of this energy. The result is that $\sim 1.5 \times 10^{15}$ water vapour molecules are condensed. Taking into account the formation of clusters, it corresponds to emission of $\sim 1 \times 10^{13}$ photons and the energy $W$ of $\sim 1 \times 10^{-2} \mathrm{~J}$ emission into the flash. But really much less quantity of photons and energy of radiation should be recorded, since a significant part of them is lost. Losses are mainly due to the absorption of water and the walls of the vessel. Also, part of the energy was preliminary liberated when the clusters were formed. In experiment [7], not more than $\sim 5 \times 10^{8}$ photons were recorded, which does not contradict to our estimation. If the transparency of the system were better, especially in the infrared and ultraviolet ranges, this number would be much larger.

\section{Comments on Plasma Models of CL/SL/LIBL}

It should be noted that the analysis of the physical processes of LIBL shows the whole inconsistency of the high-temperature models of CL/SL/LIBL. Indeed, when a primary bubble in LIBL was formed, energy not less than $\sim 10^{-1} \mathrm{~J}$ was focused in a small volume [7]. This led to the formation of plasma and caused the first flash. Part of the energy was radiated with this flash. Unfortunately, the spectrum of this radiation is not given in Ref. [7]. Further, the bubble increased in size with permanent cooling. Our estimations show that the bubble of maximum dimensions had energy of $\sim 10^{-2} \mathrm{~J}$, which is on the order of $10 \%$ of the initial energy reserve. Furthermore, up to the second flash, the bubble, at a constant pressure of $202 \mathrm{kPa}$ and constant cooling, continued to decrease in size. Consequently, with the second flash, its energy should be not more than $\sim 10^{-2} \mathrm{~J}$. But the experiment shows that the intensities of both flares are of the same order, and, consequently, the energy $\sim 10^{-1} \mathrm{~J}$ is needed to form plasma for the second flash. Where 
can the additional energy come from? Could it be a result of the collapse, as has been claimed in some papers?

If we assume that the collapse precedes the flash, what energy can it have? Unlike CL/SL, LIBL does not have any external dynamic impact that can cause collapse. This excludes the possibility of any speculation related to the concentration of the energy. Let us estimate the upper limit of the full internal energy. At point 1 , the bubble possessed the energy $\sim 1 \times 10^{-2} \mathrm{~J}$. To this energy, one should add the energy released when the volume of the bubble decreases at a constant pressure that is no more than $\sim 0.03 \times 10^{-2} \mathrm{~J}$. In sum, this gives no more than $\sim 1.03 \times$ $10^{-2} \mathrm{~J}$. Assuming that all this energy has gone to heat the vapour inside the bubble, its temperature will not be more than $10^{3} \mathrm{~K}$. We see that this temperature is not enough to explain the spectrum of CL/SL/LIBL. In fact, this temperature would have to be much lower, since with our estimates we allowed some completely absurd assumptions. The main one is that we did not take into account the cooling of the bubble between points 1 and 2, while it is this cooling that determines the rate of change of the bubble size.

\section{The CL/SL/LIBL Spectra}

\subsection{The Range of the Spectra}

Now, we refine the structure of the CL/SL/LIBL spectra. As shown in many investigations, for instance Figure 2 and Figure 5 from paper [2] and Figure 1(b) from this paper, the spectra are broad bands in the range from near infrared $(\lambda \sim$ $0.9 \mu \mathrm{m})$ to ultraviolet $(\lambda \sim 0.2 \mu \mathrm{m})$. The intensity of the radiation increases as it approaches the ultraviolet boundary. Most likely, the range is larger, and these two boundaries are defined by the absorption of the water and the walls of the vessel. In the PeTa model, the energy of the emitted photon corresponds to the condensation of the appropriate particles. Dimers and more complicated formations (clusters) can be represented as single particles. In paper [2], when evaluating the CL/SL spectra, we did not take into account the energy of the interaction of the molecules within the cluster. This approximation, which is applicable to clusters with a small binding energy of atoms/molecules, is very rough for water vapour clusters, since this energy is quite high, about $50 \%$ of the binding energy of the molecules inside the liquid. Here, we estimate the CL/SL/LIBL spectra with allowance for this energy. If the energy of the phase transition for one atom/molecule is denoted $\Lambda$, and the bound energy of the atoms/molecules in a cluster from $M$ atoms/molecules is denoted $\Gamma_{M}$ the wavelength of radiation for the cluster with $\mathrm{n}$ photon emission $\lambda_{n}^{(M)}$ follows:

$$
\lambda_{n}^{(M)}=120 \mathrm{n} / M\left(\Lambda-\Gamma_{M}\right)
$$

Equation (5) is a generalisation of Equation (5) from paper [2] when the energy of the formation of the clusters is taken into account.

The infrared boundary is defined using Equation (5) for the nonclustered molecules of water. It means that $\lambda_{1}^{1}=2.7 \mu \mathrm{m}$ for $\Lambda=44 \mathrm{~kJ} / \mathrm{mole}, n=1$, 
and $M_{I R}=1$. If one takes into account that the experimental infrared boundary $\lambda_{1}^{1}=0.9 \mu \mathrm{m}$, then the radiation from $\sim 2.7 \mu \mathrm{m}$ up to $\sim 0.9 \mu \mathrm{m}$ is absorbed by the water and the walls of the vessel. Here we do not consider the multi-photon transitions, for example $\lambda_{n \geq 2}^{1} \geq 5.5 \mu \mathrm{m}$, the possibility of which cannot be ruled out. It is important to note that without the formation of the clusters, all radiation of CL/SL/LIBL would be located in the infrared region $\lambda \geq 2.7 \mu \mathrm{m}$ (as in the formation of clouds, Figure 11 from Ref. [2]) and would be absorbed by the water. For a more detailed consideration of the problem of the clusters, we need to estimate the energy of cluster formation $\Gamma_{M}$. On the basis of numerous literature data analyses, for instance [10] and [11], we accept: $\Gamma_{M} \approx 22 \mathrm{~kJ} / \mathrm{mole}$ for $2 \leq M \leq 4 ; \Gamma_{M} \approx 23 \mathrm{~kJ} / \mathrm{mole}$ for $5 \leq M \leq 8 ; \Gamma_{M} \approx 24 \mathrm{~kJ} / \mathrm{mole}$ for $9 \leq M \leq 13 ; \Gamma_{M} \approx 25 \mathrm{~kJ} / \mathrm{mole}$ for $14 \leq M \leq 19 ;$ and $\Gamma_{M} \approx 26 \mathrm{~kJ} / \mathrm{mole}$ for $20 \leq M \leq 35$. Thus, in accordance with our model, the spectrum of the CL/SL/LIBL for water is a set of 35 bands whose centres correspond to the presented here points on the wavelength scale:

$$
\begin{aligned}
& \lambda_{1}^{(1)} \approx 2.72 \mu \mathrm{m} ; \lambda_{1}^{(2)} \approx 2.90 \mu \mathrm{m} ; \lambda_{1}^{(3)} \approx 1.90 \mu \mathrm{m} ; \lambda_{1}^{(4)} \approx 1.40 \mu \mathrm{m} ; \lambda_{1}^{(5)} \approx 1.10 \mu \mathrm{m} ; \\
& \lambda_{1}^{(6)} \approx 1.00 \mu \mathrm{m} ; \lambda_{1}^{(7)} \approx 0.86 \mu \mathrm{m} ; \lambda_{1}^{(8)} \approx 0.75 \mu \mathrm{m} ; \lambda_{1}^{(9)} \approx 0.70 \mu \mathrm{m} ; \lambda_{1}^{(10)} \approx 0.63 \mu \mathrm{m} ; \\
& \lambda_{1}^{(11)} \approx 0.57 \mu \mathrm{m} ; \lambda_{1}^{(12)} \approx 0.53 \mu \mathrm{m} ; \lambda_{1}^{(13)} \approx 0.49 \mu \mathrm{m} ; \lambda_{1}^{(14)} \approx 0.48 \mu \mathrm{m} ; \lambda_{1}^{(15)} \approx 0.44 \mu \mathrm{m} ; \\
& \lambda_{1}^{(16)} \approx 0.42 \mu \mathrm{m} ; \lambda_{1}^{(17)} \approx 10.39 \mu \mathrm{m} ; \lambda_{1}^{(18)} \approx 0.37 \mu \mathrm{m} ; \lambda_{1}^{(19)} \approx 0.36 \mu \mathrm{m} ; \lambda_{1}^{(20)} \approx 0.35 \mu \mathrm{m} ; \\
& \lambda_{1}^{(21)} \approx 0.34 \mu \mathrm{m} ; \lambda_{1}^{(22)} \approx 0.32 \mu \mathrm{m} ; \lambda_{1}^{(23)} \approx 0.31 \mu \mathrm{m} ; \lambda_{1}^{(24)} \approx 0.29 \mu \mathrm{m} ; \cdots ; \\
& \lambda_{1}^{(28)} \approx 0.24 \mu \mathrm{m} ; \cdots ; \lambda_{1}^{(35)} \approx 0.20 \mu \mathrm{m}
\end{aligned}
$$

In reality, the peaks are bands that are superimposed on each other because of their broadening, and they are not distinguished in the experiments under analysis.

It is very important to emphasize that the distance between the peaks decreases as we approach the ultraviolet boundary of the spectrum. This means that the spectral intensity of the CL/SL/LIBL $I(\lambda)=W / \delta \lambda$, where $W$ is the energy of individual peaks, has to increase even with the same magnitude of $W$. With this assumption, let's estimate the intensity increase based on the series (6):

$I(0.3 \mu \mathrm{m}): I(0.7 \mu \mathrm{m}) \approx 3.5$, that qualitatively corresponds to the experimental data (Figure 7 from Ref. [2])

In principle, the possibility of X-ray emission (not absorbed by the water) is not ruled out. But for this it is necessary to allow in a water vapour the formation of clusters containing $M \sim 400$ water molecules.

The range from $(0.9-0.7) \mu \mathrm{m}$ to $(0.3-0.2) \mu \mathrm{m}$ is experimentally observed. It corresponds to 29 peaks from $\lambda_{1}^{(7)} \approx 0.86 \mu \mathrm{m}$ to $\lambda_{1}^{(35)} \approx 0.20 \mu \mathrm{m}$.

Based on this analysis, we can conclude that if clusters are not formed, all the radiation of CL/SL/LIBL would be located in the infrared region and would be absorbed by the water. This leads to an important conclusion concerning the intensity of CL/SL/LIBL: All factors that stimulate the formation of clusters with $M$ 
$\geq 7$ during the condensation of water vapour shift the spectrum into the recorded range (near infrared, visible, and ultraviolet regions) and thus increase the intensity of CL/SL/LIBL. Thus, the cause of the increase in intensity of CL/SL/LIBL in the presence of noble gases and decreasing the temperature of the liquid is clarified. Both of these factors contribute to the formation of clusters in water vapour and increase their stability [12]. Another factor contributing to the increase in intensity at low temperatures is the small value of the equilibrium pressure of the saturating vapour. This increases the supersaturation of the vapour and, consequently, intensifies the flash (see Section 8 of this paper).

\subsection{LIBL Spectrum Features}

At LIBL under the action of the energy of the short laser impulse, the water evaporates and forms a bubble filled with a gas dissolved in water, vapour, and some amount of ions formed in the water vapour due to the dissociation of the water molecules under the action of a laser beam with respect to the reaction:

$$
\mathrm{H}_{2} \mathrm{O} \rightarrow \mathrm{H}^{+}+\mathrm{OH}^{-}
$$

Thus, the probability of the protonation of the water vapour is very high. The presence of protons in the bubble atmosphere leads to significant features of LIBL. Experimental studies based mainly on mass spectrometry measurements as well as theoretical studies of cluster ions of protonated water of type $\mathrm{H}^{+}$ $\left(\mathrm{H}_{2} \mathrm{O}\right)_{\mathrm{M}}$ and $\mathrm{D}^{+}\left(\mathrm{D}_{2} \mathrm{O}\right)_{\mathrm{M}}$ showed the existence of numerous large clusters [13]. It is interesting that the magic numbers $M=21,28,51,53$, and 55 exist, for which the clusters are very stable and, consequently, numerous. The stability of these clusters is due to the formation of clathrates. For instance, for $M=21$, the clathrate is formed with the ion $\mathrm{H}_{3} \mathrm{O}^{+}$in the centre and 20 molecules of water around him.

The main distinguishing feature of the LIBL emission spectrum from the CL and SL spectra is the presence of the intensive peak at $\lambda=0.34 \mu \mathrm{m}$ (Figure $1(\mathrm{~b})$ ). The intensity of the peak increases with the size of the bubble. Our model explains a mechanism of this peak appearance that is associated with the peculiarities described above of the formation of clusters under LIBL. We can see that the peak at $\lambda=0.34 \mu \mathrm{m}$ is associated with the emission of numerous stable clusters, with the number of molecules corresponding to the first magic number $M=21$. Indeed, in set (6) of the spectrum, line $\lambda_{1}^{(21)} \approx 0.34 \mu \mathrm{m}$. An increase in the magnitude of the peak with increasing bubble volume most likely can be explained by an increase in the probability of the formation of large magic clusters in bigger volumes which corresponds to higher laser energy. Explanation of this peak occurrence gives serious proof of the validity of the PeTa mechanism of CL/SL/LIBL.

In accordance with our model, the PeTa radiation of the LIBL has to have other features in comparison with the CL and SL. From the data of [11] [14] and [15], shown in Figure 2, it follows that in the bubble atmosphere there are small 


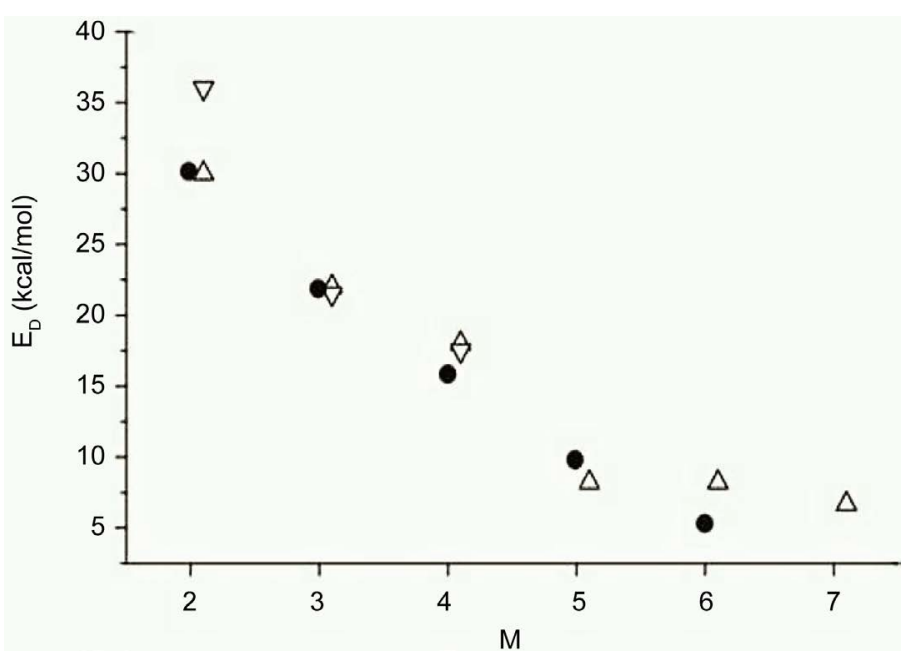

Figure 2. From Ref. [11]: Experimental values of dissociation energies of protonated water clusters versus number of molecules M; : Ref. [11]; $\Delta$ : Ref. [14]; and $\nabla$ : Ref. [15].

clusters for $M=2,3,4$, the energy of formation $\Gamma_{M}$ of which $\Gamma_{2} \approx 145 \mathrm{~kJ} / \mathrm{mole}, \Gamma_{3}$ $\approx 94 \mathrm{~kJ} / \mathrm{mole}$, and $\Gamma_{4} \approx 62 \mathrm{~kJ} / \mathrm{mole}$ is greater than the condensation energy of a large volume of water $\Lambda \approx 44 \mathrm{~kJ} / \mathrm{mole}$. This means that for these clusters, it is energetically disadvantageous to take part in the condensation process associated with the PeTa radiation: $\Lambda-\Gamma_{M}<0$. Thus, peaks corresponding to these clusters will be absent in the LIBL spectrum. The clusters must remain in the bubble atmosphere. In a very special position is a cluster of five molecules, for which $\Gamma_{5}$ $\approx 42 \mathrm{~kJ} /$ mole. In accordance with Equation (5), $\lambda_{1}^{(5)} \approx 120 / 5(44-42) \approx 12 \mu \mathrm{m}$.

Thus, the LIBL spectrum should contain a peak of radiation in a relatively far infrared region, which is absorbed with the water.

\section{The PeTa Radiation at CL/SL/LIBL}

As we have repeated many times, the evidence of the PeTa effect does not follow from general phase-transition conceptions. Recall that our first experimental studies of the PeTa effect were associated with the registration of characteristic radiation in the infrared range during the crystallisation of certain substances that are transparent in the IR range [16] [17] [18]. Our opponents deny the possibility of such radiation, and thus, high-temperature luminescence is rejected in favour of the phonon path energy removal. Here are their arguments for a case of semiconductor melt crystallisation that is very similar to our experiments [19]: "Let us consider an excited particle near a phase-transition boundary at the supercooled melt of temperature $T \approx 1000 \mathrm{~K}$. For phase-transition radiation to occur, the probability of excitation energy being converted into light emission by this particle at phase transition must be equal to or greater than the probability of the excitation energy being converted to heat. But this probability is infinitesimal. Indeed, for a free molecule in the excited state, its optical lifetime $t_{1}$ for transitions in the near-infrared range is equal to $\sim 10^{-7} \mathrm{~s}$. On the other hand, the 
non-radiative multi-phonon relaxation time $t_{2}$ is equal or less than $\sim 10^{-9} s[20]$. Then, the probability of light emission $\xi$.

$$
\xi=t_{2} / t_{1} \approx 10^{-2} \ll 1
$$

and non-radiative phase transitions have to be realized".

For existence of PeTa radiation, we need to obtain an inverse inequality

$$
\xi=t_{2} / t_{1} \geq 1
$$

Obviously, this can be done either by increasing $t_{2}$ or decreasing $t_{1}$. Let us analyse how to do it.

What is the physical meaning of $t_{2}$ ? Thus far, processes of transition from an excited state to a stable one have been investigated only for phosphors and lasers, for example, [21] [22] and [23]. Like our case, one of the main parameters is the non-radiative multi-phonon relaxation time $t_{2}$, during which the energy of the excited particle $\Delta E=E_{2}-E_{1}$ (Figure 10 from [2]) is transmitted to surrounding molecules and thus quenches the luminescence or laser effect. In solids, the non-radiative transition occurs by interaction with lattice vibrations, as well as in gases, which is most interesting for $\mathrm{Cl} / \mathrm{SL} / \mathrm{LIBL}$, it is caused as a result of inelastic collisions. For our process, the energy of the excited particle is equal to the phase-transition energy per particle:

$$
\Delta E=M\left(\Lambda-\Gamma_{M}\right)
$$

We have to understand whether in our case it is possible to increase $t_{2}$. We repeat, $t_{2}$ is equal or less than $10^{-9} \mathrm{~s}$ in solids. For greater reliability of our estimates, we take $t_{2} \approx 10^{-10} \mathrm{~s}$. The nonradiative transition involves the simultaneous emission of several phonons, which is typically required for such transitions because in most cases, the energy of a single phonon is not sufficient to match the difference in level energies. The rate of multi-phonon transitions decreases exponentially with increasing $\Delta E$ and hence an increasing of number of phonons required. As a consequence, a certain meta-stable state may exhibit a very strong augmentation in its lifetime by increasing $\Delta E$. It follows from Equation (10) that in our case, the presence of clusters instead of single atoms and molecules can solve this problem. In particular, in water vapour for a cluster of 9 molecules, $t_{2}$ increases by 4 orders of magnitude in comparison with 1 molecule, that is, $t_{2} \approx$ $10^{-6} \mathrm{~s}$. Then $\xi=t_{2} / t_{1} \geq 10>1$. Thus, in the water vapour, for clusters with the number of molecules $M \geq 9, t_{2}$ can reach a value of $10^{-6} s$, and PeTa radiation is easily realised if sufficient supersaturation has been reached.

Consequently, for clusters with $M<9$ and for single molecules, the only way to realise the PeTa effect is to decrease $t_{1}$. We accept as true the previous consideration of our opponents for a single excited particle. But first-order phase transitions can be realised only in a large ensemble of excited particles. The phenomenon under consideration seems to be similar to nuclear fission reactions or laser radiation. A critical density and number of radiators depending on the system geometry is needed for both. The state of a particle is a key circumstance in 
this case. Now, we will show that radiative phase transition would occur in our case because of Dicke's effect [9] - collective spontaneous radiation for an ensemble of excited particles. In this case, $t_{1}$ can be much less than $t_{2}$. Let us estimate under what conditions a collective spontaneous radiation can take place during first-order phase transitions. In our analysis, we will mainly follow superradiation review paper [24].

Dicke [9] showed that a system of $N$ two-level particles with a population inversion could spontaneously revert to the ground state in a time $t_{c}$ inversely proportional to the number of particles:

$$
t_{c} \sim t_{1} / N
$$

This effect occurs because a correlation is induced between the transition moments of spatially separated radiators as they interact with each other through the radiation field. As a result, the particles in a volume of macroscopic size emit coherently. Thus, the aim of our estimation is understanding if in a phase-transition system the relaxation time is $t_{c}$ and hence the following inequality has to be fulfilled:

$$
\xi=N t_{2} / t_{1} \geq 1
$$

As follows from previous estimates, this will be if the quantity of particles $N$ in the system is of order $10^{3}-10^{5}$, and thus, the radiative phase transition will be realised.

First, let us mention some peculiarities of Dicke's spontaneous radiation. A superradiation occurs because a correlation is induced between the transition dipole moments $d$ of spatially separated radiators. What is a transition dipole? A basic, phenomenological understanding of the transition dipole moment can be obtained using an analogy with a classical dipole. While the comparison can be very useful, care must be taken to ensure that one does not fall into the trap of assuming that they are the same. In the case of two classical point charges, $+g$ and $-g$, with a displacement vector $\boldsymbol{r}$ pointing from the negative charge to the positive charge, the electric dipole moment is given by $\boldsymbol{d}=g \boldsymbol{r}$. In the presence of an electric field, such as that due to an electromagnetic wave, the two charges will be exposed to a force in opposite directions, leading to a net torque $\boldsymbol{F}$ on the dipole. The magnitude of the torque is proportional to both the magnitude of the charges $g$ and the separation between them $r$. It varies with the relative angles $\theta$ of the field $E$ and the dipole $d:|\boldsymbol{F}|=g r E \sin \theta$. Similarly, the coupling between an electromagnetic wave and a transition dipole moment depends on the charge distribution within the particle, the strength of the electric field, the relative polarisations of the field, and the transition dipole moment. In addition, the transition dipole moment depends on the geometries and relative phases of the initial and final states. Thus, the superradiation effect occurs because a correlation is induced between the transition moments of spatially separated radiators as they interact with each other through the radiation field. As a result, the particles in a volume of macroscopic size emit coherently. The effect arises in 
macroscopic samples with a comparatively high concentration of preliminary excited particles. There is a minimum threshold for this concentration, and it is obvious that an increase in pressure, along with a decrease in temperature, contributes to the achievement of this threshold. The excited particles spontaneously radiate the internal energies as a short electromagnetic impulse. An increase by several orders of magnitude is found for the radiated impulse power compared to the power of non-coherent radiation of the same number of isolated particles.

At present, there is experimental evidence of superradiation effect existence for gases and activated crystals in infrared and optical ranges as well as for non-equilibrium spin systems in a radio frequency range [24]-[29].

For ordinary spontaneous emission, in which the particles decay autonomously, with a spontaneous-decay time $t_{1}$, which is independent of the number of radiators, the emission intensity $I$ is proportional to the number of radiators $N$. If $v$ is the transition frequency, the total energy $W^{(N)}$ radiated by $N$ particles is equal $N \hbar v$. The emission intensity

$$
I=W^{(N)} / t_{1}=N \hbar v / t_{1} \sim N
$$

For Dicke's superradiance, the emission intensity $I$ is proportional to the square of the number of radiators $N^{2}$ :

$$
I=W^{(N)} / t_{c}=N \hbar v / t_{c}=N^{2} \hbar v / t_{1} \sim N^{2}
$$

An effective self-induction of correlations between dipole moments can occur only if the characteristic time of this process $t_{c}$ is shorter than the relaxation time of the particle dipole moment $t_{2}$ and also shorter than $t_{1}$ (in our case, $t_{2}<t_{1}$ ).

From the standpoint of the dynamics of the excited subsystem, therefore, superradiance is a transient process that occurs over times shorter than $t_{2}$ and $t_{1}$. It has to be emphasised that this onset of correlations between radiators is an event that occurs spontaneously in the course of the emission process. This circumstance represents a fundamental distinction between superradiance and other transient coherent processes, such as the decay of free optical induction, self-induced transparency, and the photon echo, in which cases the individual radiators are in phase and the emission intensity is also proportional to $N^{2}$, but the phase coherence has been imposed by a coherent external pump [30] [31]. Thus, only superradiance is exactly our case.

The distinctive features of superradiance can be seen in an example of a typical experiment described in paper [32] (Figure 3). We will show correspondence between the processes in this superradiance system and condensation from supersaturated vapour. Let us assume that there are $N$ two-level particles in a macroscopic cylinder of length $L$ and cross area $S$, which is relatively long and open at both ends: $L \gg S^{1 / 2}$ and $V=S L$ (Figure 3 ). We specifically chose this geometry to show that, in contrast to a number of previous models of CL/SL/LIBL, spherical symmetry is not at all necessary. 


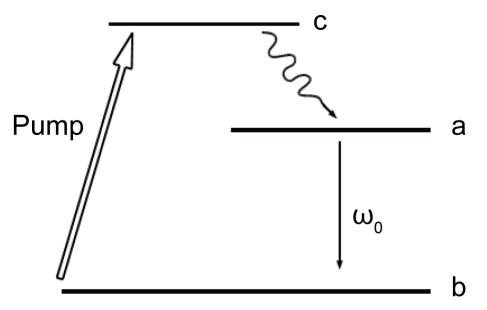

(a)

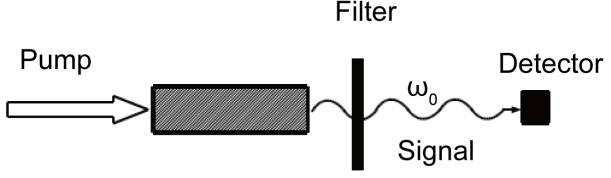

(b)

Figure 3. From Ref. [32]. A typical experiment of superradiance surveillance. (a) The energy level scheme: the ground level $-b$ with an upper excited state $-c$ and an intermediate excited level-a; (b) The scheme of the experimental setup.

Let us discuss a dimension of $V$. The effect of the shape of the sample on the dipole relaxation rate in systems with $V \ll \lambda^{3}$ was discussed in papers [33] and [34]. An attempt to apply Dicke's approach to the estimation of the superradiance effects during melt crystallisation also for volume $V \ll \lambda^{3}$ was made in paper [19]. But it is important to note that as was mentioned in paper [24], superradiance has not yet been observed in systems significantly shorter than the radiation wavelength. Our case is no exception: CL/SL/LIBL was not observed in bubbles smaller than a few microns in size. A direct proof of this statement is the experiment described in paper [35] and presented in Figure 9 from paper [2]: SL appeared only when the bubble size $2 \mathrm{R}_{0}$ exceeded $4.6 \mu \mathrm{m}$, which, with respect to series (6), fully corresponds to the infrared boundary of CL/SL/LIBL range $~ 2.9$ $\mu \mathrm{m}$. One possible reason is that dipole-dipole interactions broaden the line to the extent that the condition $t_{0}<t_{2}$, where $t_{0}$ is the delay time, may not hold for such systems.

Let us define $t_{0}$. The system of $N$ particles begins to emit at the time $t=0$. It emits a pulse whose intensity reaches a maximum $J_{\max }$ (superradiance) at the time $t_{0}$. The reason for the delay is that the decay begins with isotropic spontaneous emission, and only gradually, as the result of the interaction of particles through the radiation field, do correlations grow among dipole moments of the particles. It is at the time $t=t_{0}$ that these correlations reach their maximum. At $t_{0}$, the populations of the upper and lower working levels are equal. It follows that the number of photons in the pulse should be half the number of particles in the cloud.

If the length of the pulse is $t_{\mathcal{c}}$ then:

$$
t_{0}=t_{c} \ln N
$$

Because $N \gg 1$, we have $\ln N \gg 1$ (in a real situation, we could have $\ln N \gg 20$ ), so that the condition $t_{0} \gg t_{c}$ holds. It is thus $t_{0}$ that determines the characteristic time interval for emission of the system. Therefore, one of the conditions for superradiance is the inequality:

$$
t_{0}<t_{2}
$$

In the frame of applying this estimation to PeTa radiation, it is important to 
note that this scheme is similar to homogeneous nucleation during condensation, that is, at the beginning $(t=0)$ in the volume we have only supersaturated vapour, and all particles at the volume under consideration are excited. In this case, $t_{0}$ governs the system. This is exactly the case for CL/SL/LIBL. But if a nuclei or seed of macroscopic size exist in the system, at the beginning we have a sufficient quantity of unexcited particles on a stable level. In this case, $t_{0} \approx 0$, and $t_{c}$ governs the system. But it is not the case for CL/SL/LIBL.

Now, we repeat: One possible reason for the absence of superradiance in systems with $r_{a v}<\lambda$, where $r_{a v}$ is the average linear dimension of the system, is that dipole-dipole interactions broaden the line to the extent that the condition $t_{0}<t_{2}$ may not hold for such systems. Indeed, in a superradiative state, the dipole-dipole level width is

$$
\left(1 / t_{2}\right)_{d i p} \sim N d^{2} / \hbar r_{a v}^{3}
$$

On the other hand,

$$
1 / t_{0}=-N / t_{1} \ln N \approx\left[\left(2 \pi^{3}\right) N \ln N\right] d^{2} / \hbar \lambda^{3} \ll\left(1 / t_{2}\right)_{\text {dip }}, \text { if } r_{a v} \ll \lambda
$$

Only one previous time has it been experimentally demonstrated that superradiance may be observed in samples with dimensions comparable to the radiation wavelength. The authors of paper [36] observed the effect in the Rydberg levels of sodium atoms. The emission had a wavelength of $\lambda=1.5 \mathrm{~mm}$, and the active region had dimensions $L=5 \mathrm{~mm}$ and $S=0.75 \mathrm{~mm}^{2}$.

Thus, on the basis of experimental investigations of superradiance, we can conclude that in our case, $r_{a v}$ has to be more than maximum radiation wavelength $\lambda_{\max }$ and, as a consequence, the volume of supersaturated vapour or supercooled melt participating in the radiative phase transition has to be:

$$
V>\lambda_{\max }^{3}
$$

Now, let us return to the experiment described in paper [32]. All the particles are initially put in the upper state by a short $t_{p}$ pump pulse $\left(t_{p}<t_{c}\right)$ (Figure $4(\mathrm{a})$ ). In our case, $t_{p}=0$, corresponding to the situation that all particles (atoms, molecules, and clusters) are excited from the very beginning. The initial state of the system is non-coherent. In other words, there are no correlations between the dipole moments of the working transitions of the different particles. Then, the system of particles with a population inversion begins to decay freely, in our case homogeneous condensation. The nature of the decay depends on the relationships among the characteristic times $t_{1}, t_{2}, t_{\mathcal{O}}$ and $t_{L}=L / C$, which is the transit time of a photon through the medium ( $c$ is the speed of light).

In Dicke's original paper [9], it was shown that each particle decays independently of the others when the density of particles, $N_{V}=N / V$, is so low that the following inequality holds:

$$
t_{1}=\left(4 v^{3} d^{2} / 3 \hbar c^{3}\right)^{-1}<t_{c} \sim\left(2 \pi N_{V} v d^{2} t_{L} / \hbar\right)^{-1}
$$




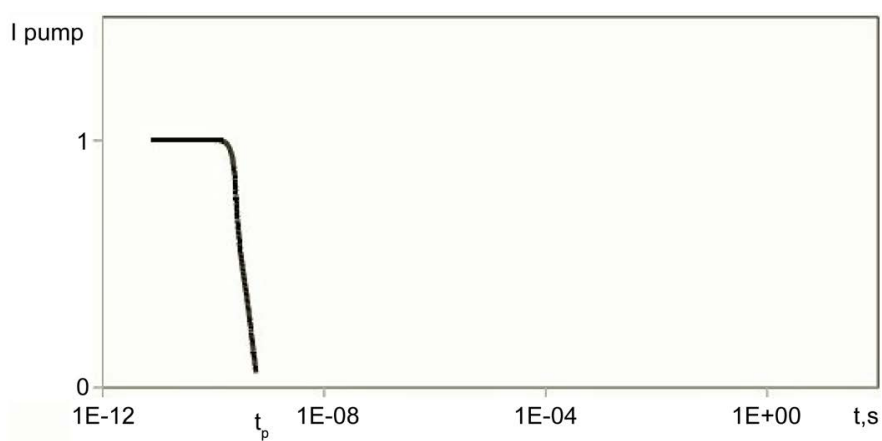

(a)

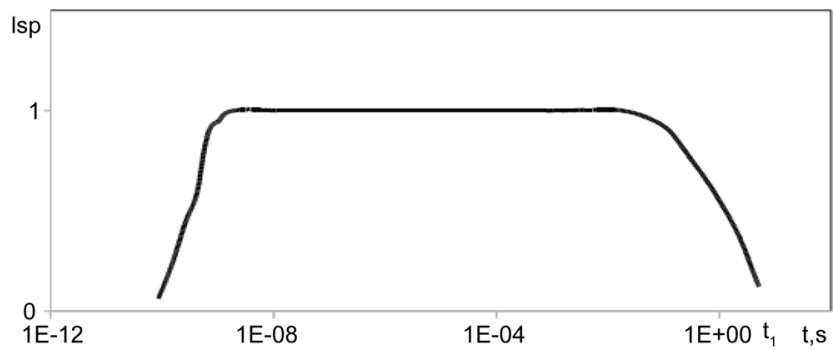

(b)

Intensity distribution

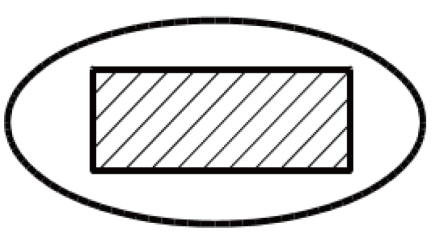

(c)

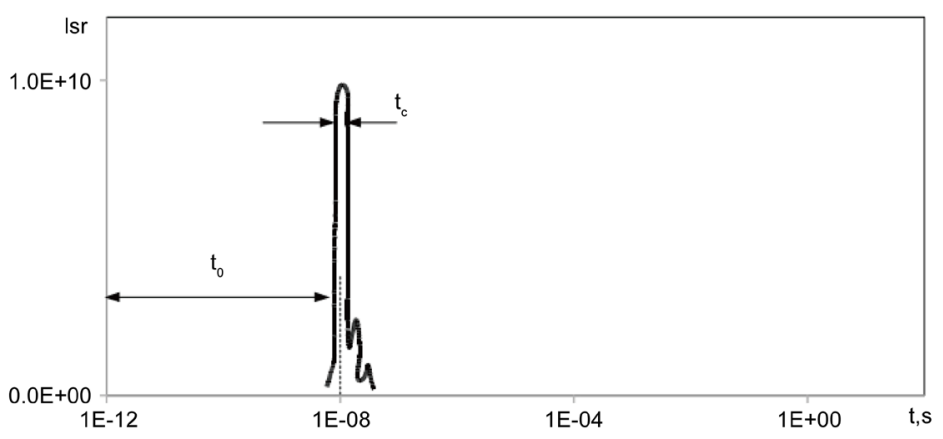

(d)

Intensity distribution

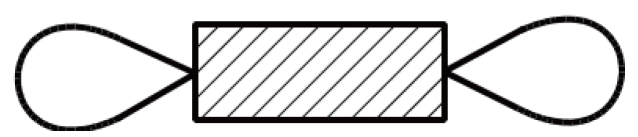

(e)

Figure 4. From Ref. [32]. Comparison of noncoherent spontaneous emission and super radiance; the time scale is logarithmic. (a) Pump pulse duration $t_{p}$, which creates a population inversion for the working transition; (b) Intensity $I_{s p}$ of emission duration $t_{1}$ in the case of noncoherent spontaneous decay-a slow exponential decay; (c) An isotropic directional distribution of the intensity; (d) The peak intensity $I_{s r}$ is roughly $10^{10}$ times $I_{s p}$, $t_{0}$ is the delay time, and $t_{c}$ is length of the pulse; (e) The observed highly directional superradiance signal (in gaseous $\mathrm{HF}$ ). 
where $d$ is the dipole matrix element of the transition. Then the energy stored in the system is radiated in a characteristic time $t_{1}$ (Figure $4(\mathrm{~b})$ ) in an isotropic fashion (Figure 4(c)).

Thus, according to Equation (20), we can obtain the minimum density of excited particles $N_{V}$ from which the superradiance can be realised.

We now assume:

$$
t_{L} \ll t_{c} \ll t_{2}, t_{1}
$$

The right-hand Inequality (21) means that the collective processes occur more rapidly than the relaxation in the individual particles. The left-hand inequality means that the photons leave the volume under consideration in a time shorter than the characteristic time for the induction of inter-particle correlations, so that stimulated processes can be ignored during superradiance. Conditions (21) determine the type of superradiance. If all these conditions hold, a system of $N$ particles will emit a superradiance pulse with a peak intensity several orders of magnitude higher than the intensity of spontaneous emission (about 10 orders of magnitude higher in the experiments from paper [32] -Figure 4(d)). Most of the energy is radiated into small solid angles along the greatest dimension of the volume (Figure 4(e)). This directionality results from the interference of the different radiators and is determined by the geometric configuration of the medium. Under the condition $t_{L} \sim t_{\mathcal{c}}$ some of the radiated energy reenters the atomic subsystem, and the emission takes the form of a train of pulses of decreasing height ("oscillatory superradiance"-Figure 4(d)).

The directionality of the superradiance, along the greatest dimension of the volume, is reminiscent of a corresponding property of the amplified spontaneous emission in mirror-free systems. Thus, under certain conditions, in a phase transition, the growth of a new phase becomes much like a cooperative optical phenomenon during which the energy of the phase transition emits as one pulse or a sequence of superradiance pulses, and the PeTa effect successfully occurs.

\section{Luminescence Pulse Duration}

Now, we can explain an interesting experimental fact: the durations of impulses in different optical ranges-red and ultraviolet (Figure 8 from paper [2]) are the same and equal to $\sim 10^{-9} \mathrm{~s}$. This fact needs special discussion. These radiations are emitted as a result of condensation of clusters containing different amounts of water molecules. The above analysis of Section 5 provides for the presence in the system of only homogeneous particles. For this reason, this model can be considered only as the first step in constructing a quantitative model of CL/SL/LIBL. But despite this, the fact of the constant duration of pulses of different frequencies finds its explanation within the framework of this model. Indeed, condensation is a collective process, and all molecules and clusters must participate in it synchronously, that is, they can all be either in the vapour phase or in the liquid one. 
The experimental data from Ref. [7], presented in Figure 5, correspond to the PeTa model. Obviously, with respect to Equation (15), the pulse width and the number of emitted photons should increase with increasing bubble size, the number of particles involved in the process. But to obtain an analytical expression of this dependence, further development of the PeTa model is necessary.

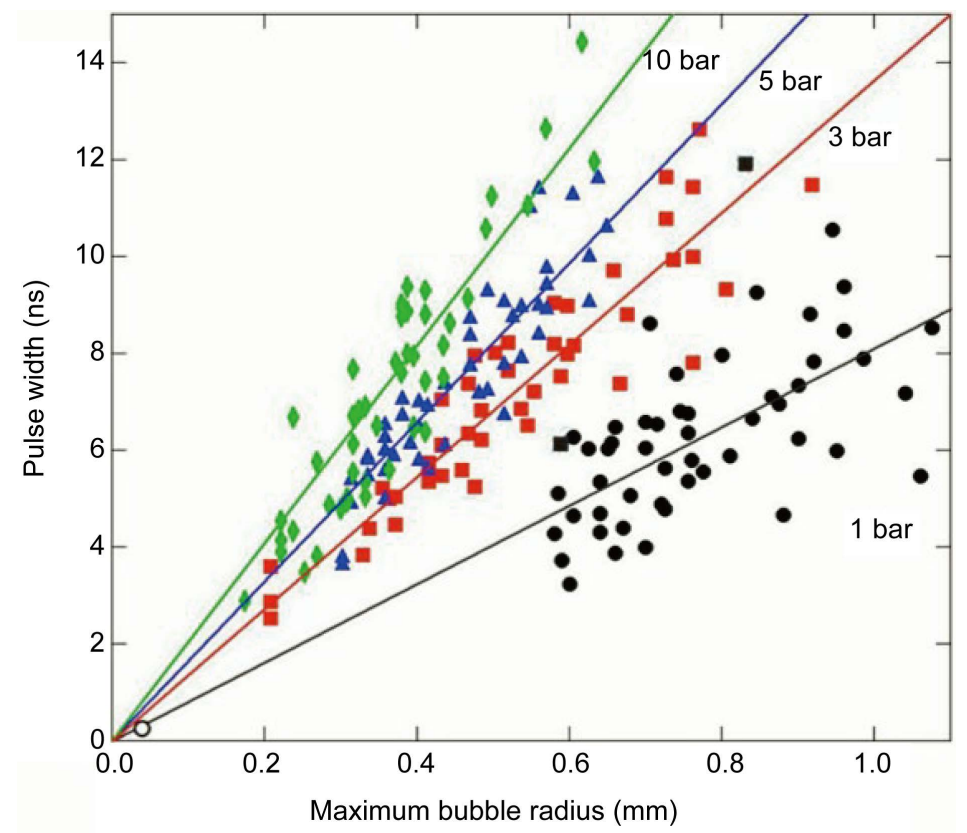

(a)

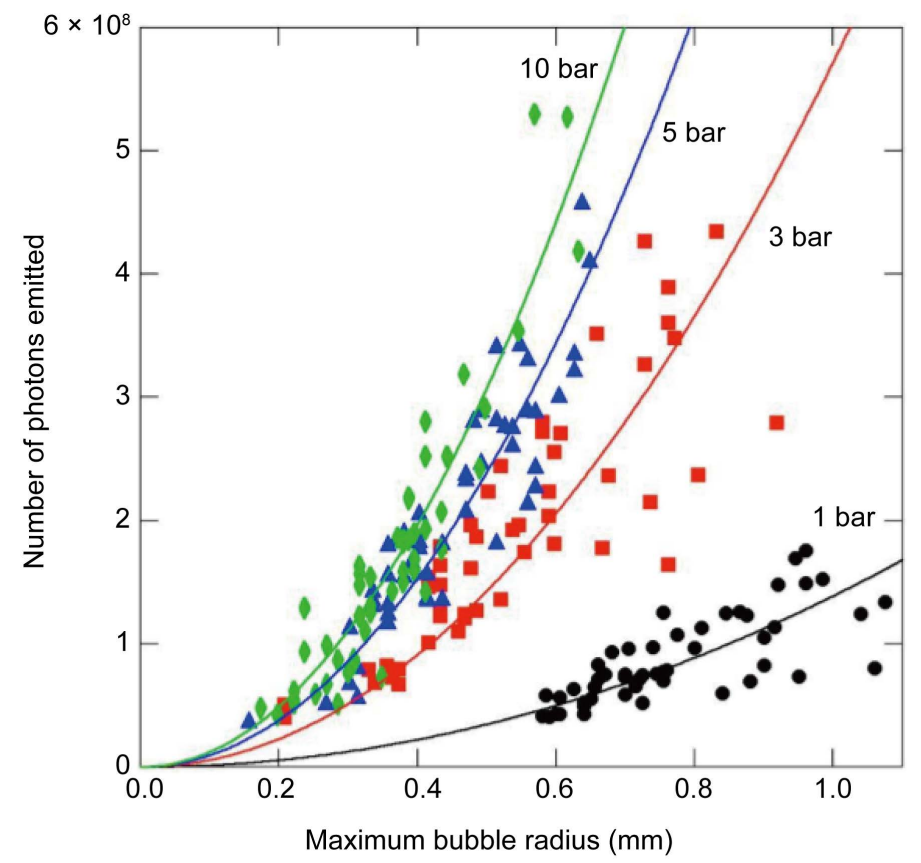

(b)

Figure 5. From Ref. [7]. (a) Luminescence pulse width as a function of the maximum bubble radius and the applied hydrostatic pressure; (b) Number of photons emitted as a function of the maximum bubble radius, with square-law fits. 


\section{The PeTa Model Explains the Main Experimental CL/SL/LIBL Data}

Table 1 presents the main experimental results concerning CL/SL/LIBL and our explanation/estimation of them on the basis of the PeTa model. As a result, today we are not aware of any experimental data that would contradict the PeTa

Table 1. The main experimental parameters of CL/SL/LIBL and estimations of them on the basis of the PeTa model.

Experimental results
CL/SL/LIBL existence.
OBSL emission has light pulses of $\sim 10^{-11} \mathrm{~s}$
duration.
LIBL emission has light pulses of $\sim 10^{-9} \mathrm{~s}$
duration, much more than CL/SL.
Every flash of OBSL/LIBL emits $10^{5}-10^{8}$
photons.
The spectra of CL/SL/LIBL are large bands
from IR, via visible, up to UV.
The spectra of CL/SL/LIBL increase the
intensity from IR, via visible, up to UV.
In LIBL, the emission peak at $0.34 \mu \mathrm{m}$ exists
on the background of the main range.
Noble gases increase CL and SL intensities.
Intensity of CL and SL increases with
decreasing liquid temperature.
Both the pulse widths in the red and the
ultraviolet spectral range are identical.

Bubble radii $R_{0}$ are in the range $\sim 2.3 \mu \mathrm{m}-2$ $\mathrm{mm}$.

Frequencies of liquid perturbations: $1 \mathrm{~Hz}$ $1 \mathrm{HHz}$; the corresponding duration of one cycle $1 \mathrm{~s}-1 \times 10^{-6} \mathrm{~s}$.

There is some, but not too much, dissolved gas; degassing on $\sim 20 \%$ from saturation.

Calibrated measurements of bubble brightness in OBSL show that each flash contains about $\mathrm{E}_{\mathrm{p}} \approx 1 \times 10^{-12}$ J energy.

MBSL has a power of $\mathrm{W}_{\mathrm{SL}} \approx 1.6 \times 10^{-8} \mathrm{~W}$ from a volume of liquid $\sim 6 \times 10^{-5} \mathrm{~m}^{3}$, excited with $1 \mathrm{~W}$ of ultrasonic energy at 24 $\mathrm{kHz}$.

Existence of other than $0.34 \mu \mathrm{m}$ emission peaks in the background of the main range.

Flash occurs $\sim 10^{-7} \mathrm{~s}$ before the minimum radius of the bubble

Correspondence to model Explanations and comments

Fully compliant with the PeTa model.

Fully compliant with the PeTa model.

Does not contradict the PeTa model.

Fully compliant with the PeTa model.

Fully compliant with the PeTa model.

Fully compliant with the PeTa model.

Fully compliant with the PeTa model.

Fully compliant with the PeTa model.

Fully compliant with the PeTa model.

Fully compliant with the PeTa model.

Fully compliant with the PeTa model.

These values are within the PeTa model.

Compliant with the PeTa model.

Fully compliant with the PeTa model.

It corresponds to the estimation for

OBSL: $\mathrm{W}_{\mathrm{OBSL}} \approx\left(10^{-7}-10^{-4}\right) \mathrm{W}$ without taking into account any absorption; for

MBSL, the number and sizes of emitting bubbles are unknown.

Does not contradict the PeTa model.

Does not contradict the PeTa model.
It is necessary to have the number of the excited particles $N \geq$ $10^{3}$ and $N / V$ more than the threshold density of them. $t_{c}$ is equal to $\sim 10^{-11} \mathrm{~s}$ if $N$ (the quantity of particles in the cloud) is $N \geq 10^{5}$.

This is due to the relatively large volume of the bubble and the large number of particles $N$.

It corresponds to $N \geq 10^{6}-10^{9}$ particles in the cloud.

The spectra are determined by the condensation of individual molecules and of clusters up to 36 molecules.

Decrease of distances between individual peaks.

Existence in the protonated vapour of a large quantity of clusters with $M=21$, the magic number of water molecules.

Noble gases form clusters with water vapour up to 60 molecules.

Two reasons: (1) the clusters in the water vapour are more stable at a low temperature; (2) it is easier to get a large supersaturation at lower temperatures.

The mechanism of light emission is the same for different wavelengths; only the quantity of molecules in the clusters is different.

Two reasons: (1) Equation (19) is fulfilled; (2) for accommodation coefficient $\alpha=0.1$, during the expansion of the bubbles, these radii give a volume for the evaporation of liquid that is sufficient for $N \approx 10^{7}$ particles in the cloud.

For accommodation coefficient $\alpha=0.1$, during expansion of the bubbles, these frequencies give time for the evaporation of liquid that is sufficient for $N \approx 10^{7}$ particles in the cloud.

It gives a necessary pressure ratio of the gas and vapour in the bubble.

Our estimation gives $\mathrm{E}_{\mathrm{p}} \approx\left(1 \times 10^{-10}-1 \times 10^{-12}\right) \mathrm{J}$ of energy;

energy absorption by the water and the walls of the vessel has to be taken into account.

The absorption of radiation by liquid and glass or quartz must be taken into account.

It is likely that their presence is due to the excitation of gases and other substances dissolved in the liquid; their excitation occurs under the influence of shock waves occurring in the liquid.

After the flash, a collapse occurs and then the bubble reaches a minimum size. 
model, and we continue to assert that there is no mystery to the CL/MBSL/ OBSL/LIBL phenomena, as well as no reason to hope that they can be used for high-temperature chemical reactions, and even more so for a thermonuclear ones.

\section{PeTa Radiation at Different Temperatures}

In this section, we examine some experimental data concerning the influence of the temperature of the environment on the intensity of the PeTa radiation [37] [38] [39]. Of course, the scheme of the experiment does not correspond to the CL/SL/LIBL, but the experiment shows that the intensity of the PeTa radiation can increase with decreasing temperature of the environment. For the experiments, a PerkinElmer Frontier MIR spectrometer was used, with a DynaScan interferometer cooled with a liquid nitrogen MCT detector and sealed and desiccated Ge-coated $\mathrm{KBr}$ optics, which enabled a scan range of $\Delta \lambda=1.2-28 \mu \mathrm{m}$. An irradiative object, a $200 \mathrm{ml}$ plastic cup, was placed approximately $4 \mathrm{~cm}$ from the input window of the spectrometer.

The surface of the cup was moistened with water to facilitate condensation and the precipitation vapour upon cooling. A horizontal line before point A1 (Figure 6) presents integral intensity of the IR radiation $\mathrm{U}_{2}$ from the surface of the cup at the beginning of the experiment. Obviously, this is the equilibrium Planck's radiation corresponding to room temperature. Several seconds after beginning the measurements, a similar cup with liquid nitrogen was placed inside the first cup (Figure 6, point A1). The integral intensity of the radiation immediately decreased (Figure 6, the time between points $\mathrm{A} 1$ and B1) as a result of the temperature decreasing to $77 \mathrm{~K}$. Ice (evidently $\mathrm{H}_{2} \mathrm{O}$ and most likely $\mathrm{CO}_{2}$ ) appeared on the cup surface and the integral intensity of the radiation increased (Figure 6, the range between points B1 and C1). In the paper [37], it was shown that this is the PeTa radiation. While liquid nitrogen is inside the radiating cup, the intensity of the radiation is conserved (Figure 6, the range between points $\mathrm{C} 1$ and D1). When the liquid nitrogen is removed, the intensity immediately decreases (Figure 6, the range between points D1 and F1). We repeated the procedure many times (6 times in Figure 6), and each time the situation was repeated.

When we changed the plastic cup with an ice cup, and learned to adjust and measure the temperature of the radiating cup, we obtained a temperature dependence of the integrated intensity of the PeTa radiation (Figure 7).

\section{CL/SL/LIBL Is One of a Number of Cooperative Phenomena Caused by the PeTa Effect}

It is important to emphasize that CL/SL/LIBL occupies the place in a number of other cooperative phenomena caused by the PeTa effect, for instance [1] [3] [4] [5] [16] [17] [18] [37] [38] [39]. Let us make an additional digression that has no direct relationship to the $\mathrm{CL} / \mathrm{SL} / \mathrm{LIBL}$ but is very interesting for the illustration 


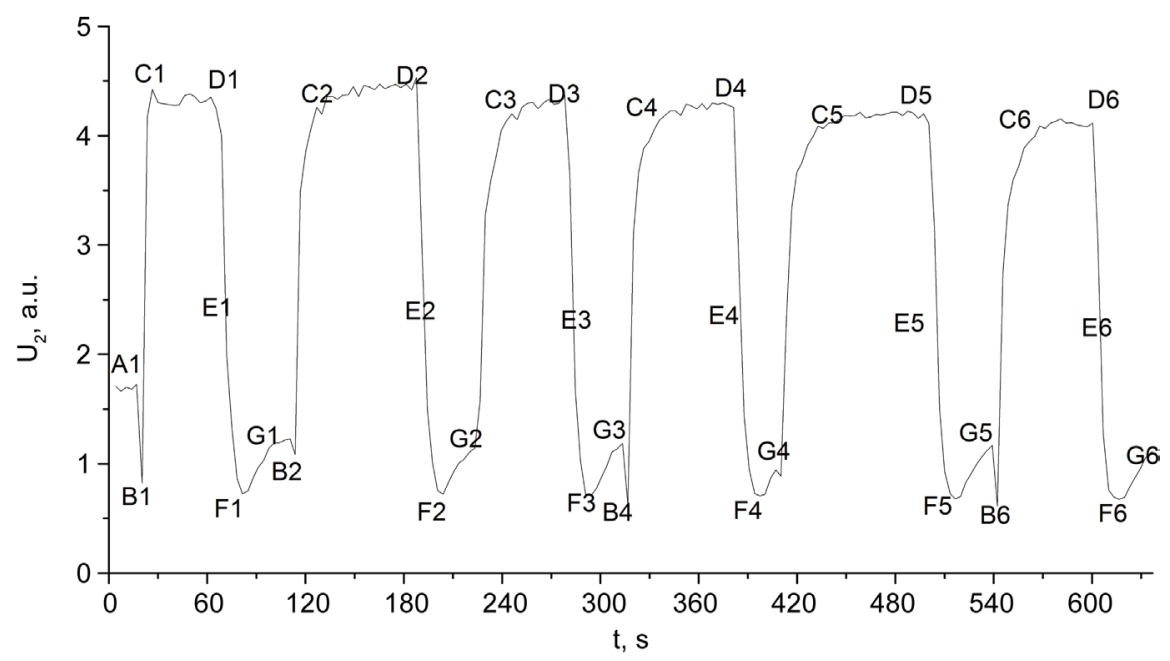

Figure 6. From Ref. [37]: Time dependence of integral intensity of the IR radiation $U_{2}$ (arbitrary units) between 4.0 and $22 \mu \mathrm{m}$ from a wet plastic cup.

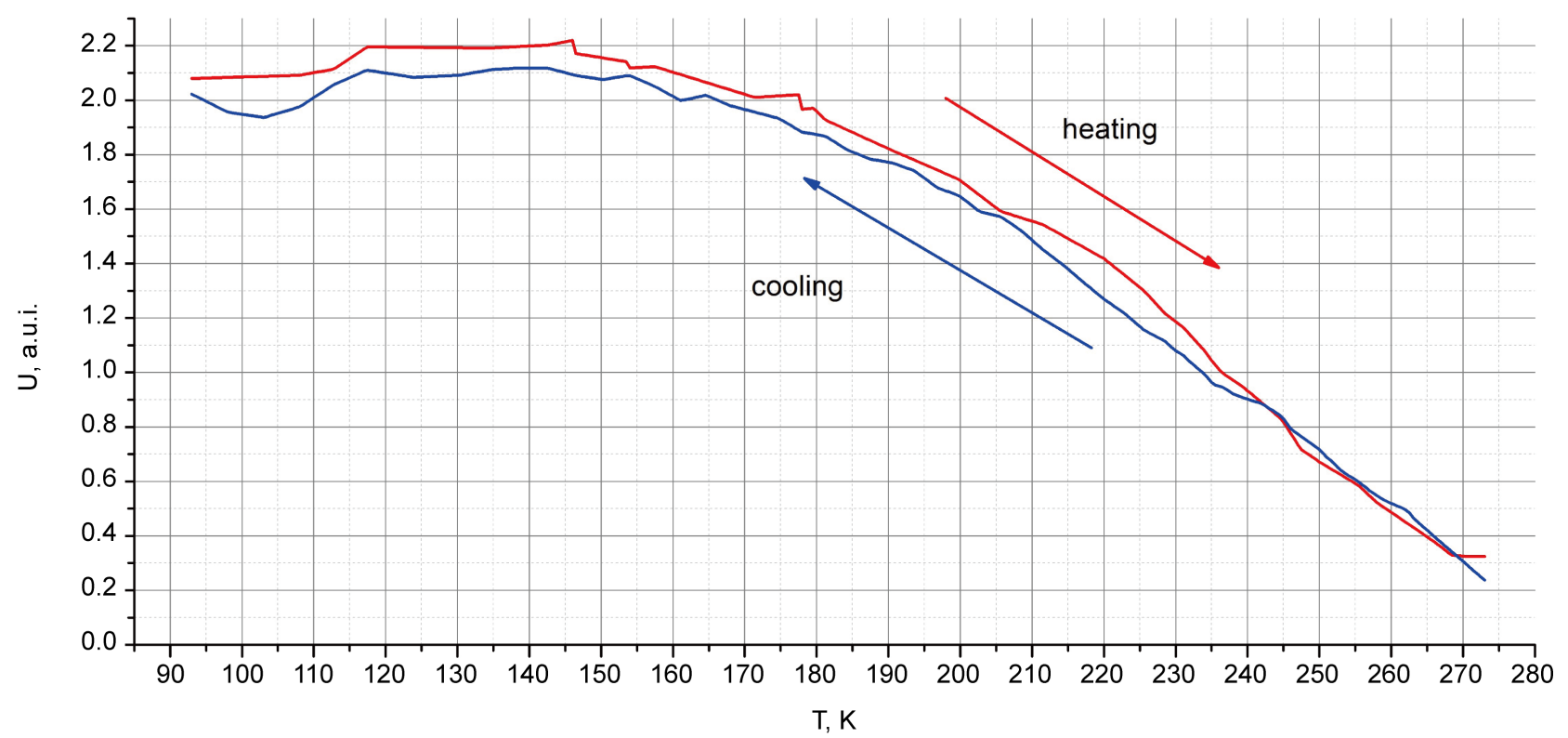

Figure 7. From Ref. [39]: Temperature dependence of integral intensity of the IR radiation U (arbitrary units) from an ice cup.

of the PeTa effect application to a wide range of similar physical phenomena. The classical theory of melt crystallisation suggests that the maximum growth rate is observed when, at some temperature, an optimum relationship between the supercooling of the melt and its viscosity is set. Actually, for the majority of substances, the crystal growth rate turns out to be well above the theoretical value and, in addition, does not vary over a wide temperature range [40]. This inconsistency was still unexplained. In the framework of the PeTa model, it finds a natural explanation. Indeed, if the phase transition is radiative, the growth rate considerably increases and the temperature, supercooling, and viscosity at the interface are governed not by external conditions, but by the transport of radiation inside the crystal. 


\section{Conclusions}

In this paper, a model of cavitational luminescence (CL), sonoluminescence (SL), and laser-induced bubble luminescence (LIBL) developed on the basis of the PeTa effect is improved. For the first time, this model was presented in paper [2]. In the model, the main role is given to the liquid in which the cavitation occurs. The evaporation and subsequent condensation of the liquid inside the bubble are responsible for the LIBL/CL/SL. Apparently, the dissolved gases and other impurities in the liquid are responsible for peaks that appear in the background of the main spectrum. They are most likely excited by a shock wave that occurred during cavitation. This model requires more precise future analysis, but already in this version, the main experimental results have revealed a qualitative and semiquantitative explanation.

What is the main value of this model? We have already mentioned in paper [2] that the colour and power of the PeTa radiation are determined by the type of vapour, pressure, temperature, intensity of mixing, and position of the emitting layer. Thus, the model gives us information on the physical processes in many natural systems through the colours and power of the radiation.

\section{References}

[1] Ravilous, K. (2010) Cloud Power. New Scientist, 208, 38-41. https://doi.org/10.1016/S0262-4079(10)62951-X

[2] Tatartchenko, V.A. (2017) Sonoluminescence as the PeTa Radiation. Optics and Photonics Journal, 7, 27-55. https://doi.org/10.4236/opj.2017.72004

[3] Perel'man, M.E. and Tatartchenko, V.A. (2007) Phase Transitions of the First Kind as Radiation Processes. 1-17. arXiv:0711.3570

[4] Perel'man, M.E. and Tatartchenko, V.A. (2008) Phase Transitions of the First Kind as Radiation Processes. Physics Letters A, 372, 2480-2483. https://doi.org/10.1016/j.physleta.2007.11.056

[5] Tatartchenko, V.A. (2009) Some Peculiarities of First Order Phase Transitions. Reviews on Advanced Materials Science, 20, 58-69.

[6] Ayad, M. (1971) Electromagnetic Radiation during Ultrasonic Cavitation. Infrared Physics, 11, 249. https://doi.org/10.1016/0020-0891(71)90027-3

[7] Bruian, E.A. (2013) Luminescence from Cavitation Bubbles. Bulletin of the Transilvania University of Braşov, Series I: Engineering Sciences, 6, 27-32.

[8] Buzukov, A.A. and Teslenko, V.S. (1971) Sono-luminescence Following Focusing of Laser Radiation into a Liquid. JETP Letters, 14, 189-193.

[9] Dicke, R.H. (1954) Coherence in Spontaneous Radiation Processes. Physical Review, 93, 99-110. https://doi.org/10.1103/PhysRev.93.99

[10] Lebedev, N.G. (2011) The Stable Geometry Configurations of the Ground and Perturbed States of the Water Dimer. Bulletin of Volgograd State University, Series 1, Mathematical Physics, No. 1, 113-119.

[11] Wroblewski, T., Ziemczoneka, L., Gazdaa, E. and Karwasza, G.P. (2003) Dissociation Energies of Protonated Water Clusters. Radiation Physics and Chemistry, 68, 313-318. https://doi.org/10.1016/S0969-806X(03)00307-4

[12] Khodorkovsky, M.A., Artamonova, T.O., Murashov, S.V., Michael, D., Rakcheeva, 
L.P., Belyaeva, A.A., Timofeev, N.A., Melnikov, A.S. and Shakhmin, A.L. (2007) Investigation of the Composition of a Mixture of Water Vapor with Argon by the Mass Spectrometry Method of a Supersonic Molecular Beam. Journal of Technical Physics, 77, 16-23.

[13] Annika, L. (2009) Theoretical Investigations of Water Clusters, Ice Clathrates and Functionalized Nanoparticles. Ph.D. Thesis, Linköping University, Linköping.

[14] Magnera, T.F., David, D.E. and Michl, J. (1991) The First Twenty-Eight Proton Hydration Energies. Chemical Physics Letters, 182, 363-370.

[15] Honma, K., Sunderlin, L.S. and Castelman Jr., A.W. (1993) Guided-Ion Beam Studies of the Reactions of Protonated Water Clusters, $\mathrm{H}\left(\mathrm{H}_{2} \mathrm{O}\right)^{+}{ }_{\mathrm{n}}(\mathrm{n}=1-4)$, with Acetonitrile. The Journal of Chemical Physics, 99, 1623. https://doi.org/10.1063/1.465331

[16] Tatarchenko, V.A. (1979) Appearance of Distinguishing Features in Emission Spectra during Crystallization of Substances Transparent in the IR Region. Soviet Physics-Crystallography, 24, 238-239.

[17] Umarov, L.M. and Tatarchenko, V.A. (1984) Differential Spectra of Crystallization Radiation of Alkali-Metal Halides. Soviet Physics-Crystallography, 29, 670-673.

[18] Tatarchenko, V.A. and Umarov, L.M. (1980) Infrared Radiation Accompanying the Crystallization of Sapphire. Soviet Physics-Crystallography, 25, 748-749.

[19] Sall, S.A. and Smirnov, A.P. (2000) Phase Transition Radiation and the Growth of a New Phase. Technical Physics, 45, 849-853. https://doi.org/10.1134/1.1259737

[20] Karlov, N.V. (1988) Lectures on Quantum Electronics. Nauka, Moscow. (In Russian)

[21] Riseberg, L.A. and Moos, H.W. (1968) Multi-Phonon Orbit-Lattice Relaxation of Excited States of Rare Earth Ions in Crystals. Physical Review, 174, 429-438.

[22] Layne, C.B., Lowdermilk, W.H. and Weber, M.J. (1977) Multi-Phonon Relaxation of Rare-Earth Ions in Oxide Glasses. Physical Review B, 16, 10-20.

https://doi.org/10.1134/1.1259737

[23] Petrov, V., Peterman, K., Griebner, U., Peters, V., Liu, J., Rico, M., Klopp, P. and Huber, G. (2006) Continious-Wave and Mode-Locked Lasers Based on Cubic Sesquioxide Crystalline Hosts. Proceedings of SPIE, 6216, 62160H.

[24] Andreev, A.V., Emel'yanov, V.I. and Il'inski, Yu.A. (1980) Collective Spontanous Emission (Dicke's Superradiation). Soviet Physics Uspekhi, 23, 493-514.

[25] Andreev, A.V., Emel'yanov, V.I. and Il'inski, Yu.A. (1988) Cooperative Phenomena in Optics. Science, Moscow.

[26] Andreev, A.V. (1990) Optical Super-Radiance: New Ideas and New Experiments. Soviet Physics Uspekhi, 33, 997-1020.

[27] Milonni, P.W. (1976) Semi-Classical and Quantum-Electro-Dynamical Approaches in Non-Relativistic Radiation Theory. Physics Reports (Section C of Physics Letters), 25, 1-81.

[28] Zheleznyakov, V.V., Kocharovskii, V.V. and Kocharovskii, V. (1989) Polarization Waves and Super-Radiance in Active Media. Soviet Physics Uspekhi, 32, 835-870. https://doi.org/10.1070/PU1989v032n10ABEH002764

[29] Men'shikov, L.I. (1999) Super-Radiation and Related Phenomena. Soviet Physics Uspekhi, 169, 113-154.

[30] Allen, A. and Eberly, J.H. (1975) Optical Resonance and Two-Level Atoms. Wiley-Interscience, New York. 
[31] Golenishchev-Kutuzov, A. and Samartsev, V.V., Eds. (1975) Electromagnetic Super-Radiance. Tatgosizdat, Kazan. (In Russian)

[32] Scribanowitz, N., Hermann, I.P., MacGillivray, I.C. and Feld, M.S. (1973) Observation of Dicke Superradiance in Optically Pumped HF Gas. Physical Review Letters, 30, 309-312.

[33] Friedberg, R., Hartmann, S.R., and Manassah, J.T. (1972) Influence of Resonant Frequency Shifts on Superradiant Damping. Physics Letters, A5, 365-368. https://doi.org/10.1016/0375-9601(72)90533-6

[34] Friedberg, R. and Hartmann, S.R. (1974) Super-Radiant Stability in Specially Shaped Small Samples. Optics Communications, 10, 298-301.

[35] Ketterling, J.A. and Apfel, R.E. (1998) Experimental Validation of the Dissociation Hypothesis for Single Bubble Sonoluminescence. Physical Review Letters, 81, 4991-4994.

[36] Gross, M., Goy, P., Fabre, C., Haroche, S. and Raimond, J.M. (1979) Maser Oscillation and Microwave Superradiance in Small Systems of Rydberg Atoms. Physical Review Letters, 43, 343-346.

[37] Tatartchenko, V.A., Smirnov, P.V. and Wu, Y. (2013) First Order Phase Transitions as Radiation Processes. Optics and Photonics Journal, 3, 1-12. https://doi.org/10.4236/opj.2013.38A001

[38] Tatartchenko, V.A., Smirnov, P.V. and Jin, H. (2014) First Order Phase Transitions as Radiation Processes, Part Two. Optics and Photonics Journal, 4, 26-37. https://doi.org/10.4236/opj.2014.42005

[39] Tatartchenko, V.A., Smirnov, P.V. and Wu, Y. (2014) PeTa Radiation under Ice Deposition. 13th International Conference on the Physics and Chemistry of Ice, 17-20 March 2014, Hanover, Poster A19.

[40] Vollmer, M. (1939) Kinetik der Phasenbildung. [Kinetics of Phase Formation.] Steinkopff, Dresden. 